\title{
Impact of Multiple Frequency Channels Usage on the Performance of TDMA-based Broadband Fixed Cellular Multihop Networks *
}

\author{
Imran Syed*, Mohamed H. Ahmed ${ }^{* *}$, Halim Yanikomeroglu*, Samy Mahmoud* \\ *Broadband Communication and Wireless Systems Centre (BCWS), \\ Department of Systems and Computer Engineering, Carleton University, Ottawa, Canada \\ ** Faculty of Engineering and Applied Science, Memorial University, St. John's, Canada \\ imran@sce.carleton.ca, $\underline{\text { mhahmed@engr.mun.ca, }}$ halim@sce.carleton.ca, $\underline{\text { mahmoud@ @sce.carleton.ca }}$
}

\begin{abstract}
This paper analyzes the impact of having more than one frequency channel on outage probability and node throughput in TDMA-based broadband fixed cellular multihop networks. The scenario considered is a low node density, start-up network with inadequate single-hop coverage. In this scenario the user terminals themselves act as relays, therefore, there are no additional relays deployed by the service provider; besides, no separate relaying channels are used (this scenario ensures a fair comparison between the multihop cellular network considered here and the traditional single-hop cellular network). In a situation where there is outage with a single-hop due to poor coverage, a suitable multihop route with minimum number of hops is sought. It is observed that as the number of available channels increases the coverage is enhanced remarkably; in addition, the average node throughput is increased as well although no explicit attempt for throughput maximization is made during the search for the multihop routes.
\end{abstract}

Keywords-Multihop; Relaying; Mesh Network; Fixed Wireless; Cellular; TDMA.

\section{INTRODUCTION}

Growing wireless multimedia applications are demanding high data rates and varying QoS requirements. To provide this at affordable price to customers, service providers are looking for systems that require low deployment cost and which can accommodate as many high data rate users as possible. Therefore need has arise to look into alternate wireless network architectures other than the traditional cellular networks to achieve the above objectives. However these new architectures will be really appealing if flexible enough to couple with or support the existing globally deployed cellular network.

Multihop (MH) network is one such architecture in which users in the network with good links to the base station (BS), apart from their own communication, support other users. Hence high data rate coverage comes from the users and not necessarily the base stations, as in the case of traditional cellular networks. The concept of multihopping is not new [1], and is the basis of ad-hoc networks, an infrastructure-less architecture. However there is lot to be explored and understood about its performance in infrastructure-based networks like cellular. Some studies on performance evaluation of multihopping have shown promising results in both fixed networks [2] and mobile cellular networks [3]; yet a great deal of investigation is to be done from different perspectives to build confidence on the viability and performance gains from $\mathrm{MH}$ implementation. Some obvious issues associated with $\mathrm{MH}$ communication are: increased data latency, more battery drain, complex routing and channel assignment schemes, control signaling overheads, sophisticated and thus costly user terminals etc. Implementation of $\mathrm{MH}$ architecture in fixed wireless network is also referred to as Wireless Mesh Networks. Some work on mesh network in LMDS bands is available [2], however the restriction of line of sight (LOS) is a prime implementation barrier. Our work is based on standard drafted by IEEE 802.16 for fixed wireless access in 2-11 GHz band, in which LOS requirement is not the concern. A characteristic of $\mathrm{MH}$ is that, it needs an additional channel for relaying, which means more use of scarce and costly frequency spectrum. Some salient features of $\mathrm{MH}$ network considered in this paper are:

- Highly aggressive frequency reuse of 1 .

- No separate relays or relaying channels.

- "Minimum number of hops route first" route assignment policy.

- Directional, switched beam antennas at BSs and user nodes as well.

The objective of this paper is to investigate the impact of available number of frequency channels on the network performance improvement brought by multihopping in a SH network with poor coverage. The performance metrics investigated are outage probability and node throughput. These are evaluated with respect to average generated node traffic level. The results are compared with traditional SH

\footnotetext{
* This research is funded by National Capital Institute of
}

Telecommunications (NCIT), Ottawa, Canada 
cellular network.

\section{SYSTEM MODEL}

\section{A. Network Architecture}

The fixed cellular $\mathrm{MH}$ network considered in this study is shown in Figure 1. A noise limited, low user density network is considered representing a start-up network. Although most studies on multihop networks consider more than two network entities [3][5] like BS, user nodes, access points or mesh insertion points and wireless router etc; our study is focused on only two main entities typical of any cellular network, namely, BSs and user nodes. This means no new network infrastructure, easy implementation, and integration of multihopping through modified software algorithms. The analysis focuses on the uplink transmission. Frequency reuse of 1 is considered meaning no cell planning required. Synchronous TDMA has been considered. It means that all node transmissions on the uplink are concurrent and slot synchronized. Simple retransmit ARQ scheme is used to take care of high frame outage links. Both, user nodes and BSs have directional, switched beam antennas. The network entity terminologies used are defined below:

Source Node: The user node from which the data transmission is initiated.

Relayee: The user node in need of relaying assistance.

Relay: The user node which apart from its own communication, is forwarding other nodes traffic.

\section{B. Path Loss Model}

The following large scale path loss $(P L)$ model is considered.

$$
20 \log \left(\frac{4 \pi d_{o}}{\lambda}\right)+10 n \log \left(\frac{d}{d_{o}}\right)+X_{\sigma}
$$

where $X_{\sigma}$ is the lognormal shadowing parameter with zero mean and standard deviation of $\sigma$. Multipath fading is assumed to be averaged out (by antenna micro-diversity or some other means).

\section{Routing}

Abundant literature is available on $\mathrm{MH}$ routing especially in ad-hoc networks, and some in cellular networks [4], which can be adapted as needed. In this work, we used a simple yet optimal route search scheme, in order to find multiple $\mathrm{MH}$ routes for each node-BS pair. The cost chosen is large scale path loss (PL), rather than signal to interference-plus-noise ratio (SINR). The reason is, since all user nodes are fixed the PL will be unchanged on all links. Thus we can develop a $\mathrm{MH}$ routing table for each node-BS pair. These routing tables need not to be updated frequently, and will be modified only when new nodes will register in the network. In contrast to PL; SINR is a dynamic quantity and will vary from slot to slot, thus we will have to resort to on-the-spot iterative search if we use it as the cost for route selection. Moreover, since the network is noise limited, therefore PL is a good measure of SINR. Pre-built routing tables can save lot of processing time, which would be required for on-the-spot iterative search of the optimum available route. Moreover, since we are doing burst level simulation, i.e. every new burst arrival at a certain source node may be assigned a different route based on the availability, therefore such on-the-spot iterative route search may not be an efficient solution at high traffic densities and may lead to undesirable set-up and processing delays. The criterion used for setting up (ordering) the multiple routes for any node-BS pair is

$$
\min \left(\max \left(P L_{i}\right)\right), i=1,2, \ldots, m .
$$

where $m$ is the total number of hops.

All links with path loss greater than a certain path loss threshold $\left(P L_{t h}\right)$ are rejected during the route search procedure. Only 2 and 3-hop routing tables are developed for each node-BS pair. The reason for using an exhaustive (but simple) search algorithm rather than any widely used fast converging algorithms like Djkstra's or Bellman-Ford algorithms is simply that, although these algorithms are efficient in providing the optimal route, when used to find multiple alternate routes with constraint on number of hops, they turned out to be more complex than the exhaustive search. However, more investigation can be done in this area.

\section{Algorithm for Routes Search}

Step 1: Reject node-node and node-BS links with $P L>P L_{t h}$. Step 2: List all 2-hop and 3-hop routes between source node and BSs.

Step 3: Arrange in an ascending order, the routes found in step 2 using the criterion $\min \left\{\max \left(P L_{i}\right)\right\}$, where $m=2$ and 3 . Step 4: If there is a tie in $\max \left(P L_{i}\right)$, then the route with the least $\left\{\Sigma P L_{i}\right\}$ is put on top.

\section{Route Selection / Slot(s) Assignment Policy}

The route assignment policy used in this research is "minimum number of hops route first". Algorithm of route selection is as follow: First the source node looks for a possible $\mathrm{SH}$ connection to $\mathrm{BS}(\mathrm{s})$ with required free slots available, if not possible it explores all 2-hop routes in the routing table, followed by 3-hop routes; if, even a 3-hop route is not found, the burst is denied service. Once the issue of free slots on a particular route is resolved, these slots are checked for the threshold signal to interference plus noise ratio $\left(S I N R_{t h}\right)$, if the achieved signal to interference plus noise ratio $\left(S I N R_{a c h}\right)$ is less than $S I N R_{t h}$ on any hop of the candidate route, this route is rejected and the next best route 
in the table is explored. Once a route is selected, the best SINR free slot on every hop is assigned to the requested connection in order to maximize the throughput. This joint route selection and slot assignment scheme is relatively simple to implement and consumes less spectral resources (time slots or channels).

\section{Fixed Transmit Power / Adaptive Coding and Modulation}

Fixed transmit power $\left(\mathrm{P}_{\mathrm{t}}\right)$ is considered on each hop. Although this may lead to higher interference levels, we exploit the high $\mathrm{P}_{\mathrm{t}}$ and good SINR links, whenever possible, to achieve higher node throughput using bit-interleaved coded-modulation (BICM). Table 1 shows this mapping for $\mathrm{BER}=10^{-6}$. Buffered transmission with regenerative (digital) relaying is considered. Buffering gives the flexibility of independently choosing coding rate and modulation level on each hop based on its SINR and simple call admission control policy, at the expense of increased hardware cost.

Table 1: Identification of the adaptive coding and modulation level from SINR (at BER $=10^{-6}$ ).

\begin{tabular}{|c|c|c|}
\hline SINR $(\mathrm{dB})$ & $\mathrm{bps} / \mathrm{Hz}$ & $\begin{array}{c}\text { Adaptive Coding } \\
\text { and Modulation }\end{array}$ \\
\hline$>26.0$ & 6 & $1-64$ QAM \\
\hline $21.94-26.0$ & 5.25 & $7 / 8-64$ QAM \\
\hline $19.0-21.94$ & 4.5 & $3 / 4-64$ QAM \\
\hline $17.7-19.0$ & 4 & $2 / 3-64$ QAM \\
\hline $15.0-17.7$ & 3.5 & $7 / 8-16$ QAM \\
\hline $14.02-15.0$ & 3 & $3 / 4-16$ QAM \\
\hline $12.0-14.02$ & 2.67 & $2 / 3-16$ QAM \\
\hline $10.93-12.0$ & 2 & $1 / 2-16$ QAM \\
\hline $7.45-10.93$ & 1.5 & $3 / 4-$ QPSK \\
\hline $4.65-7.45$ & 1 & $1 / 2-$ QPSK \\
\hline$<4.65$ & 0 & - \\
\hline
\end{tabular}

\section{SIMULATION PARAMETERS AND ASSUMPTIONS}

A network of four square shaped cells, each of size $3 \times 3 \mathrm{~km}^{2}$ is considered, with 200 nodes uniformly distributed in the entire network. Multiple frequency carriers at 2.5 $\mathrm{GHz}$ and channel bandwidth of $5 \mathrm{MHz}$ each are considered. The system noise figure is $5 \mathrm{~dB}$. Both, BSs antennas and user nodes antennas are switched beam type with $30^{\circ}$ beam width. Node-BS links have propagation exponent of 3.8, while Node-Node links have 4. Zero mean lognormal shadowing with $\sigma_{s}=4 \mathrm{~dB}$ and $6 \mathrm{~dB}$ are considered on NodeBS links and Node-Node links respectively. Reference distance, $d_{o}=10 \mathrm{~m}$. Fixed transmit power of 2 watts is used on all hops. Network traffic is bursty in nature with a Poisson distributed arrival. Burst size is exponentially distributed with mean of $15 \mathrm{Kbits}$. TDMA with 10 slots/channel is used. Bursts are served in the form of frames at the rate of 100 frames/sec. Each burst is assigned one slot per frame on each hop of the entire route. Snap shot analysis is done on frame level.

\section{SIMULATION RESULTS}

\section{A. Outage Probability}

In this paper, outage is the aggregate effect of poor coverage (due to inadequate SINR) and blocking (due to lack of time slot resources). As shown in Fig. 2, SH network due to poor coverage $(\sim 62 \%)$ has consistently high outage irrespective of the number of available channels. However if channels are few like in the case of two channels, blocking also starts contributing to the outage probability at high traffic levels as illustrated in Fig. 3. The strength of $\mathrm{MH}$ to provide high coverage is obvious from Fig. 2, where we are getting 2-4 orders of magnitude improvement (reduction) in outage probability, depending upon the number of available channels. More the channels available, more profound is the improvement over the range of generated node traffic levels. This is because when more channels are available, the source node and relay(s) can avoid interference, especially in the presence of directional antenna, to find a good $\mathrm{MH}$ route to the destination, thus ensuring high coverage. This is true even when we have no separate relaying channels and fixed $P_{t}$. The contribution of multihopping in reducing outage is presented in Fig. 4.

\section{B. Net Node Throughput}

Fig. 5 compares the average net node throughput of $\mathrm{SH}$ and $\mathrm{MH}$ networks with respect to an ideal network. In an ideal network, all the generated node traffic is supported hence there is a linear relationship of a unity slope between the average net throughput and average generated traffic. In SH network, any traffic generated from poorly covered nodes is just not supported, hence a lower slope of approximately 0.62 for light to medium generated traffic levels, irrespective of the number of available channels. However, as traffic grows, the impact of resource limitation (channels) comes into play resulting in degradation of node throughput. In MH network, full coverage and high spectral efficiencies (Fig. 6) due to short links, leads to a $100 \%$ delivered throughput for light-medium traffic levels. Later, at high traffic levels, increased interference leads to degraded spectral efficiency (Fig. 6) and channel exhaustion, resulting in relatively lower throughput but still superior to corresponding $\mathrm{SH}$, except for 2-channel case. The high data rate coverage provided by multihopping is extended to high traffic levels as number of channels increases.

\section{CONCLUSION AND OBSERVATIONS}

From the above study, it is found that, for a traditional fixed cellular network in the start up phase with low upfront infrastructure and poor coverage, implementing multi- 
hopping even without additional relaying channels or relays, provides not only significant coverage boost but also high node throughput. This superior performance of $\mathrm{MH}$ is found to be directly related to the number of available frequency channels in the system. More the frequency channels, more the performance improvement from multihopping, even at high traffic levels. Proper selection of cell sizes based on node density, to ensure reasonable number of alternate $\mathrm{MH}$ routes, can be an important initial decision in a successfully feasible implementation. Our next research target is to incorporate route selection schemes and power control, which will aim throughput maximization (in addition to coverage enhancement).

\section{Acknowledgement}

We would like to thank Dr. Lek Ariyavisitakul for providing Table 1 on BICM.

\section{REFERENCES}

[1] Y.-D. Lin and Y.-C. Hsu, "Multihop cellular: A new architecture for wireless communication", IEEE INFOCOM 2000, pp. 1273-1282.

[2] T. Fowler, "Mesh performance in finite spectrum", white paper v1.0.doc, Radiant Broadband Wireless Access Network, 2001; available online at www.radiantnetworks.com.

[3] V. Sreng, H. Yanikomeroglu, and D. Falconer, "Coverage enhancement through two-hop relaying in cellular radio systems", IEEE WCNC 2002, vol. 2, pp. 881-885.

[4] R. Ananthapadmanabha, B. S. Manoj, and C. S. R. Murthy, "Multihop cellular networks: the architecture and routing protocols", IEEE PIMRC 2001, vol. 2, pp. G78-G82.

[5] W. Zirwas, M. Lampe, H. Li, M. Lott, M. Weckerle, and E. Schulz, "Multihop communications in future mobile radio networks", IEEE PIMRC 2002, vol. 1, pp. 54-58.

[6] V.Erceg et.al, "An empirically based path loss model for wireless channels in suburban environments", IEEE JSAC, vol. 17, no. 7, pp. 1205-1211, July 1999.

[7] V.Erceg et.al, "Channel Models for Fixed Wireless Applications", IEEE 802.16.3c-01/29r4

[8] T. S. Rappaport, Wireless Communication: Principles and Practices, Prentice Hall PTR, 1996, chapter 3, pp. 106-108. 


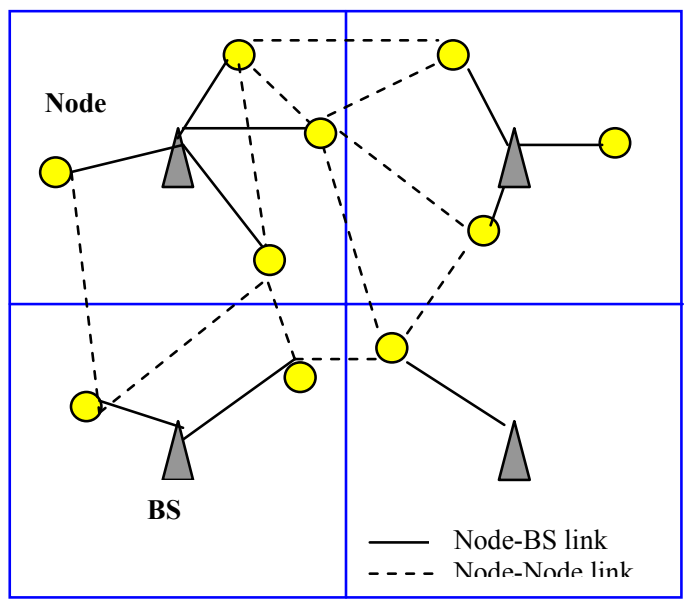

Figure 1. Multihop network model

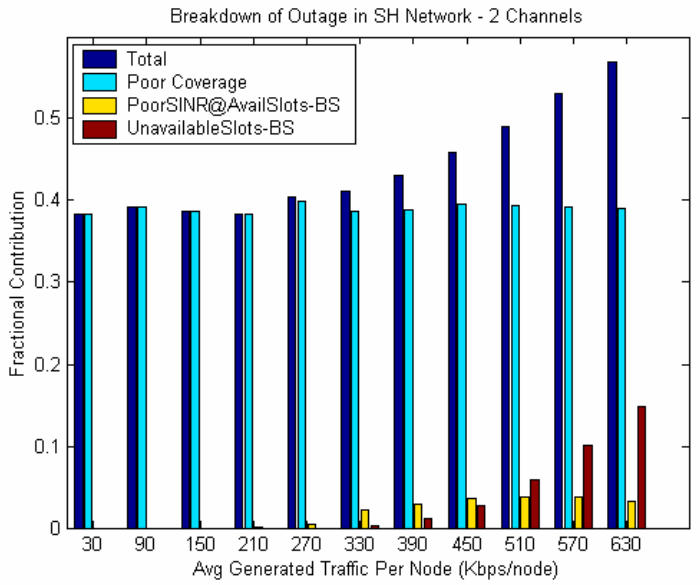

Figure 3. Contribution of different factors in outage probability of single-hop network with 2-channels

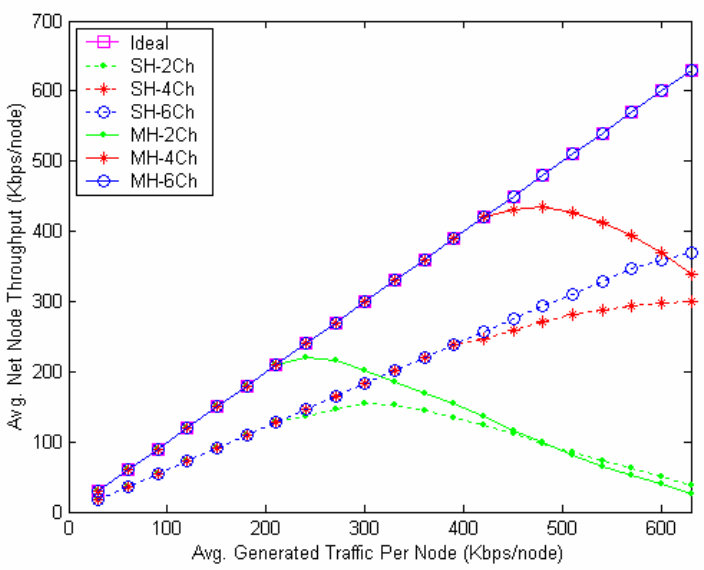

Figure 5. Average net node throughput of single-hop and multihop networks with 2, 4 and 6 frequency channels

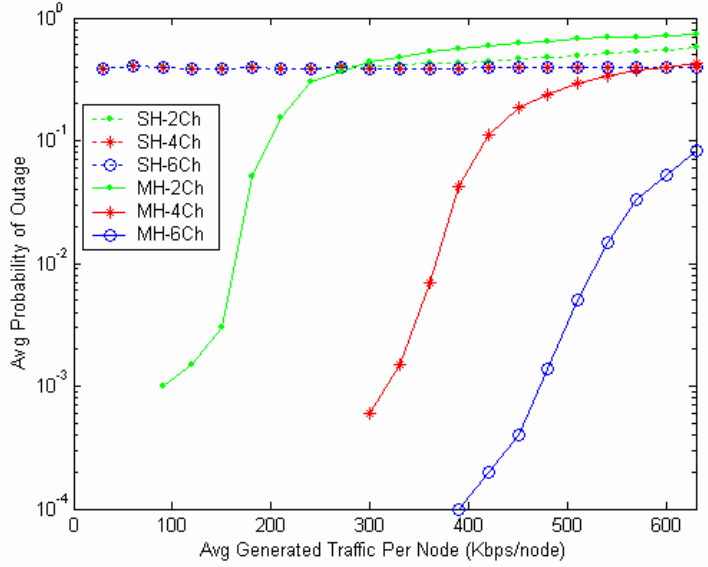

Figure 2. Average probability of outage in single-hop and multihop networks with 2, 4 and 6 frequency channels

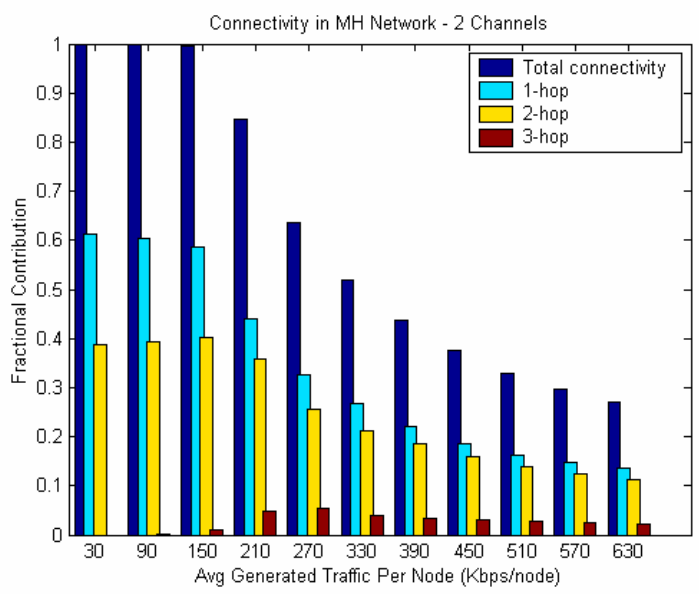

Figure 4. Contribution of $\mathrm{m}$-hop connectivity in multihop network with 2-channels

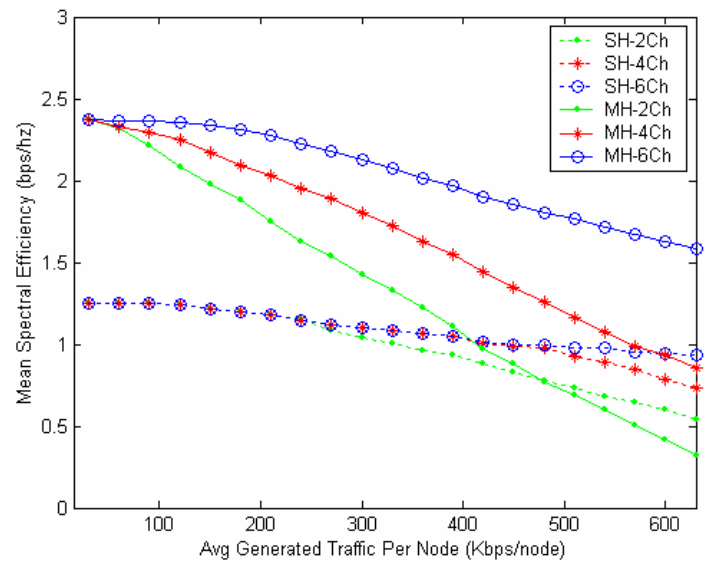

Figure 6. Mean spectral efficiency of single-hop and multihop networks with 2, 4 and 6 frequency channels 